\title{
Landmine detection by 3D GPR system
}

\section{Motoyuki Sato, Yuya Yokota, Kazunori Takahashi, Mark Grasmueck}

Motoyuki Sato, Yuya Yokota, Kazunori Takahashi, Mark Grasmueck, "Landmine detection by 3D GPR system," Proc. SPIE 8357, Detection and Sensing of Mines, Explosive Objects, and Obscured Targets XVII, 835710 (10 May 2012); doi: 10.1117/12.918517

Event: SPIE Defense, Security, and Sensing, 2012, Baltimore, Maryland, United States 


\title{
Landmine detection by 3DGPR system
}

\author{
Motoyuki Sato*a $^{\text {a }}$, Yuya Yokota ${ }^{\mathrm{a}}$, Kazunori Takahashi ${ }^{\mathrm{a}}$, Mark Grasmueck ${ }^{\mathrm{b}}$ \\ ${ }^{a}$ Center for Northeast Asian Studies, Tohoku University, Sendai, 980 8576Japan; \\ ${ }^{\mathrm{b}}$ RSMAS, University of Miami, 4600 Rickenbacker CSWY, Miami, FL 33149 USA
}

\begin{abstract}
In order to demonstrate the possibility of Ground Penetrating Radar (GPR) for detection of small buried objects such as landmine and UXO, conducted demonstration tests by using the 3DGPR system, which is a GPR system combined with high accuracy positing system using a commercial laser positioning system (iGPS). iGPS can provide absolute and better than centimetre precise $\mathrm{x}, \mathrm{y}, \mathrm{z}$ coordinates to multiple mine sensors at the same time. The developed " $3 \mathrm{DGPR}$ " system is efficient and capable of high-resolution 3D shallow subsurface scanning of larger areas (25 $\mathrm{m}^{2}$ to thousands of square meters) with irregular topography . Field test by using a 500MHz GPR system equipped with 3DGPR system was conducted. PMN-2 and Type-72 mine models have been buried at the depth of 5-20cm in sand. We could demonstrate that the 3DGPR can visualize each of these buried land mines very clearly.
\end{abstract}

Keywords: GPR, 3DGPR, Landmine, Humanitarian Demining, iGPS

\section{INTRODUCTION}

High spatial resolution is required for GPR (Ground Penetrating Radar) to be used for buried mine detection, UXO detection. If we need classification of detected objects and confirmation for buried mines, the resolution must be subcentimetre precision. The spatial resolution of GPR system is determined by several factors which include radar range resolution and azimuth resolution. The radar range resolution is given by frequency bandwidth of the radar system. On the other hand, the azimuth resolution of GPR is related to a few factors. The most important factor for azimuth resolution is GPR data acquisition density. However, it is not simple, but it will also be controlled by the radar range resolution and the area size of the data acquisition.

Conventionally, GPR data acquisition was conducted as follows. 1-D survey ( data acquisition) is carried out by moving a GPR system along a straight survey line. The data acquisition density is determined by the spacing of data acquisition along the survey line. In order to record the position of data acquisition, a wheel attached to the system is typically used. 2-D survey is carried out by acquiring the data on 2-dimensional grid. In order to acquire the data in 2-SD form, normally we set multiple parallel survey lines and acquire the 1-D data, and create 2-D data sets. If we need high-density data sets, the interval of survey line spacing must be small.

There is not very clear criterion which determines the required data acquisition density along the survey line and the spacing of survey lines. There is not much problem in acquiring very high density data sets along a survey line, because most of the commercial GPR system can acquire data at a few hundred cycles, and even if the GPR moves $1 \mathrm{~m} / \mathrm{s}$, data density can be $1 \mathrm{~cm}$, which is much shorter than $1 / 10$ wave length at $500 \mathrm{MHz}$ centre frequency. And even if we increase the data acquisition rate, the survey time along the same length of a survey line does not change. On the contrary, if we decrease the spacing between the survey lines, we can decrease the data acquisition density across the survey lines, but it will increase the data acquisition time, for a certain area under the survey, because it simply increases the number of survey lines. Data acquisition time is very important factor in field survey, and unfortunately, it is a conflicting factor to the high azimuth resolution, as mentioned above.

*sato@cneas.tohoku.ac.jp; phone +81 22795 6075; fax +81 227956074

Detection and Sensing of Mines, Explosive Objects, and Obscured Targets XVII,

edited by J. Thomas Broach, John H. Holloway Jr., Proc. of SPIE Vol. 8357, 835710

(c) 2012 SPIE · CCC code: 0277-786X/12/\$18 - doi: 10.1117/12.918517

Proc. of SPIE Vol. $8357835710-1$ 
Another factor which we have to consider is the position control accuracy for moving the GPR system along the survey line. In normal GPR surveys, we set a measuring tape along the survey line, and a GPR system is moved along the tape. The system position can be controlled accurately, if the system moves on a flat surface such as a concrete pavement, however, if it is on a rough surface such as grass land, the position accuracy must be worse than a few $\mathrm{cm}$, and it decreases the GPR profile quality. However, it was not serious problem, if we acquire along single survey line, because the inaccurate position information does not affect to the GPR image quality, because the slight move of the image position along the survey line cannot be recognized in the GPR profile. However, it causes serious decrease of the data quality if we use 2-D GPR survey. If we set multiple 1-D GPR profiles, slight miss-positioning in each 1-D GPR profile enlarges the error in 2-D GPR profile. For example, the image of a straight buried pipe cannot be imaged as a straight line.

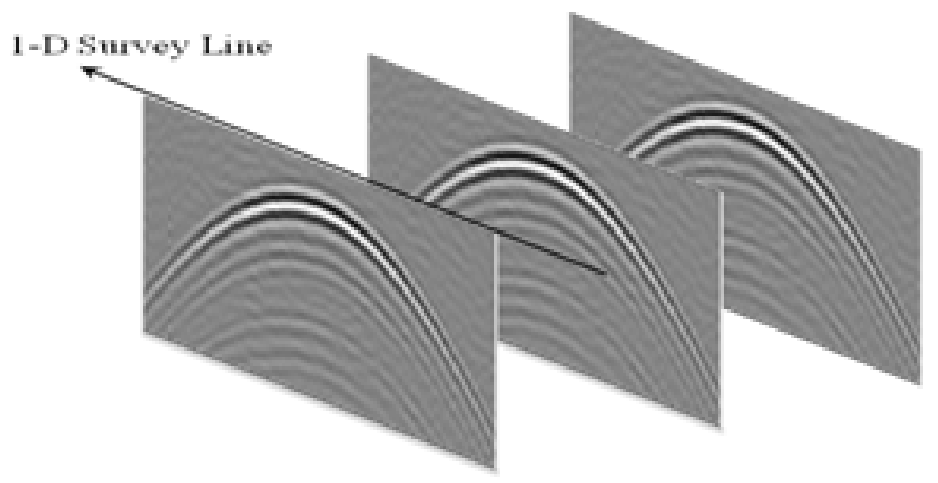

Figure 1(a) 2-D regular survey lines

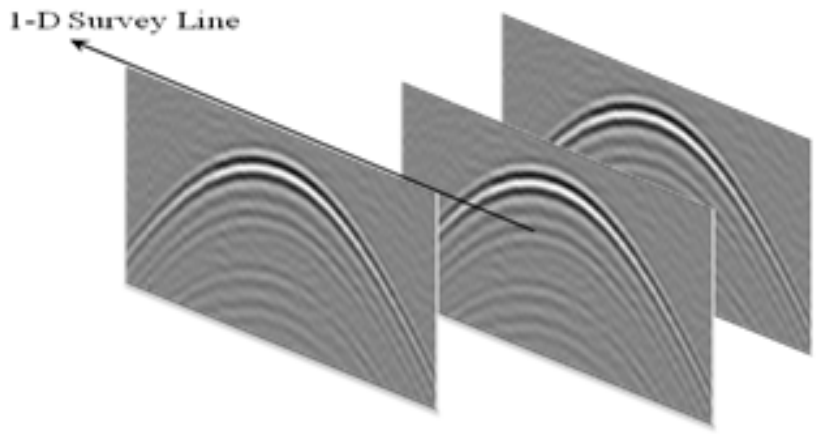

Figure 1(b) 2-D irregular survey lines

In order to control the position of the GPR system along the survey line, we need a sophisticated system. One of the solutions is the use of a robotic arm. In 2006, we developed two GPR systems mounted on an unmanned buggy with a robotic arm[1][2][3]. One of them is SAR-GPR system, which is a array type GPR system developed by Tohoku University, mounted on an unmanned Mine Hunter Vehicle(MHV), developed by Chiba university and Fuji heavy Industry Co. as shown in Fig.2. SAR-GPR demonstrated that it can re-construct high resolution GPR images thanks to the high accuracy positing by the robotic arm, which is shown in Fig.3.

The other system is ALIS mounted on Gryphon shown. An unmanned buggy system can survey over a large area, and improves the work efficiency based on conventional metal detection. The robot arm uses a GPR system "ALIS", which was developed by Tohoku University, that allows users to remotely confirm the presence of mines. ALIS was equipped on a robot of a buggy system Gryphon shown in Fig.4 developed by the research group at Tokyo Institute of Technology. All the same hardware and software of ALIS were used, and the data acquisition rate can be improved by the scanning with a robot arm. The buggy mounted ALIS uses a VNA based GPR with a Vivaldi antenna, which gives the best GPR performance. Fig.5 shows an example of GPR images acquired and processed by ALS on Gryphon. Thanks to the very accurate positioning of the sensor by a robotic arm, we can obtain very clear GPR images. One buried anti-personnel mine can be seen in this GPR horizontal profile. 


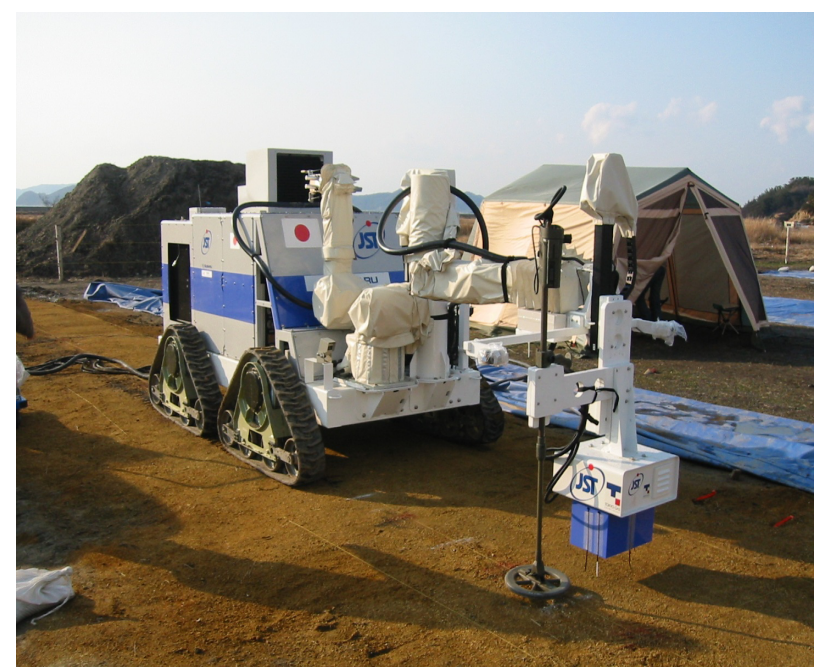

Figure. 2. SAR-GPR mounted on Mine Hunter Vehicle.

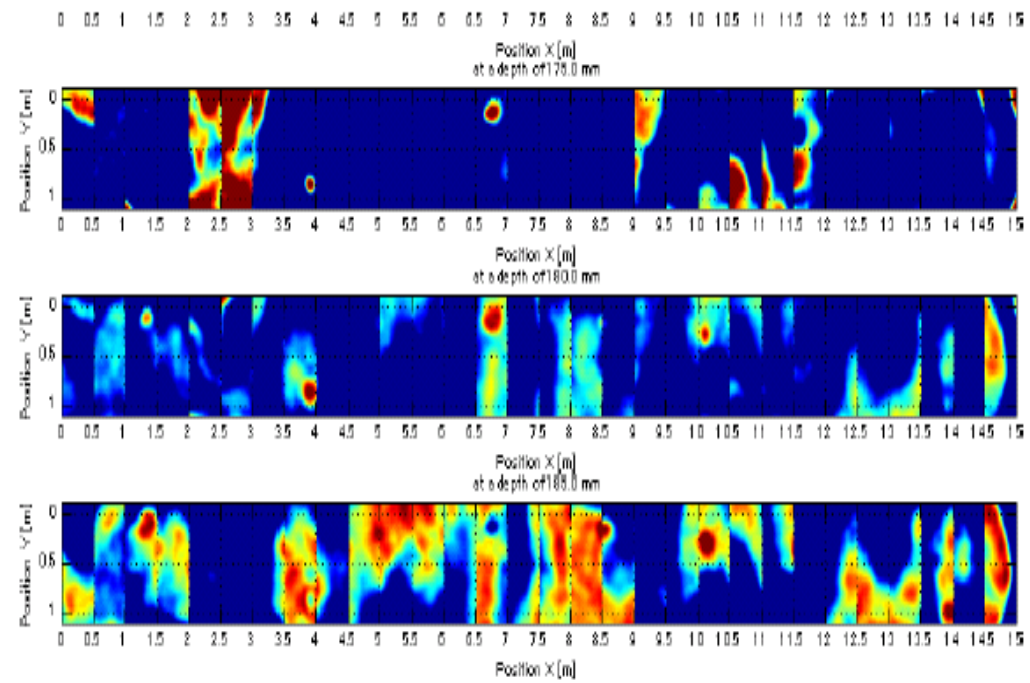

Figure3. SAR-GPR horizontal image. Three figures shows horizontal slice of GPR images at 135, 180 and $185 \mathrm{~mm}$. We can find the images of mines at different depths. 


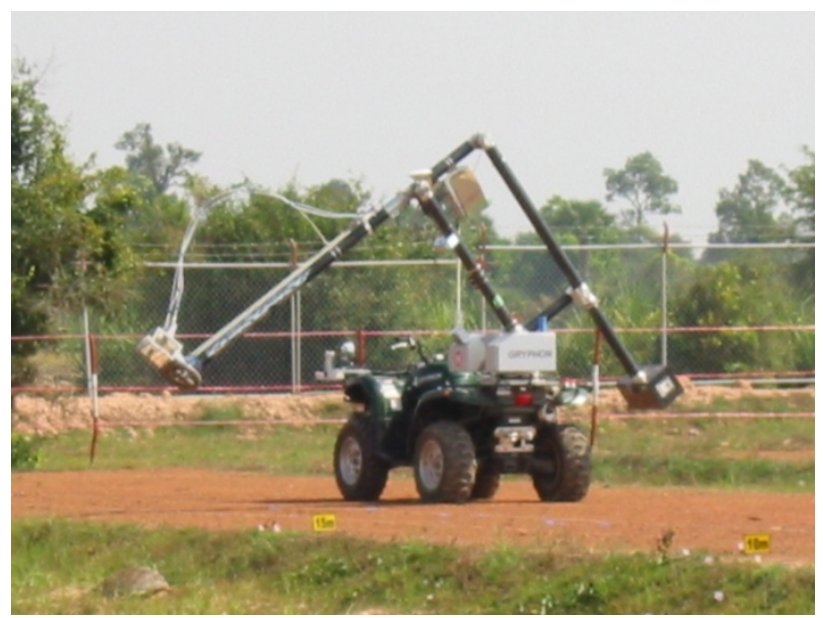

Figure 4. ALIS mounted on a buggy Gryphon (developed by Tokyo Institute Technology). A Vivaldi antenna is attached with a metal detector sensor on the robot arm. The buggy mounted ALIS uses a VNA based GPR for the best performance.

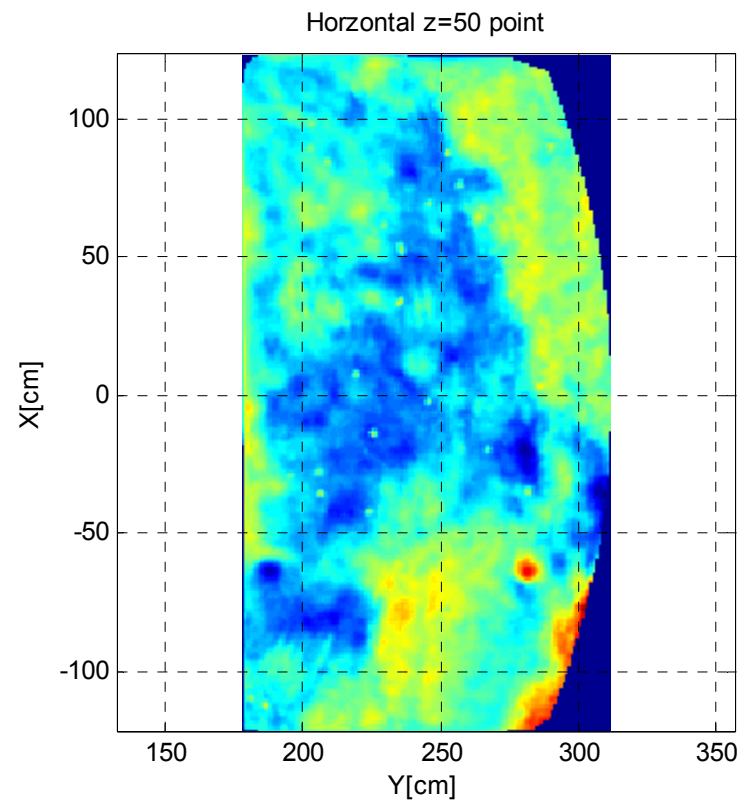

Figure 5. ALIS GPR horizontal slice acquired by Gryphon.

Although these GPR systems could demonstrate very accurate and high-resolution GPR images, which are quite suitable for land mine detection, we think this approach is complicated and not easy to use in general conditions, especially in humanitarian demining activities.

Tohoku University has developed a dual sensor system "ALIS", which is a hand-held GPR[4] . ALIS uses a CCD camera for antenna position tracking with an image processing algorithm, and can provide visualized image. ALIS has been deployed in Cambodia and has demonstrated its capability of detecting buried anti-personnel mines with GPR. However, ALIS always uses information obtained from an Electromagnetic Induction sensor (Metal Detector) and GPR together, and if only from the GPR image is not easy to identify buried landmines.

Tohoku University and University of Miami are collaboratively working on the application of 3DGPR for detection of buried explosive devices[5]. Currently we are investigating the use of iGPS, a large work volume metrology method, as a complementary tracking device for the robotic arm scanned GPR system. iGPS can provide absolute and better than centimetre precise $\mathrm{x}, \mathrm{y}, \mathrm{z}$ coordinates to multiple mine sensors at the same time. At University of Miami we have 
developed a novel 3DGPR system for efficient and high-resolution 3D shallow subsurface scanning of larger areas (25 $\mathrm{m}^{2}$ to thousands of square meters) with irregular topography [8].

In this paper, we introduce a high-precision 2-D GPR imaging by using 3DGPR system, and demonstrate the applications to detection and imaging of buried land mines.

\section{3DGPR SYSTEM}

The basic idea of 3DGPR, which has been developed by Grasmueck [5] is virtual realization of high-accurate position controlling of the GPR system. Instead of moving the GPR system along a straight survey line very accurately, we move the system roughly along survey lines, but the spacing of the survey lines is much smaller than normal survey. In addition, the positing of the GPR system is measured by iGPS system very accurately. After the data was acquired on the "rough" 2-D survey grid, the data was re-arranged on the "accurate" 2-D grid by 3-D interpolation as shown in Fig.6. By this signal processing, we can obtain very accurate 2-D GPR data sets.

In order to obtain the high accurate position of the GPR system, we use a laser positioning system. This is a commercial system (Nicon iGPS) . We set at least two transmitters, which transmit a potion signal of the transmitter by modulated laser signal. A received set on the GPR system receive the laser signals and decode the signal. More than two received signals are used to calculate the position of the receiver, and it can be estimated at sum millimeter accuracy. The GPR system which is used in the 3DGPR does not require any specific characteristics, and we use a commercial GPR system (RAMAC GPR) in this study. The position information acquired by iGPS and the GPR data acquired by RAMAC GPR must be synchronized, and small modifications of the hardware must be done.

High density 2-D GPR data sets must be acquired to process the data to obtain the accurate virtual 2-D GPR data. 3-D data interpolation (2-D GPR acquisition position, and GPR signal in the time-domain create the 3-D data sets), and we obtain the accurate 3-D GPR data sets. The 3-D GPR data sets can be processed and migration images can also be obtained.

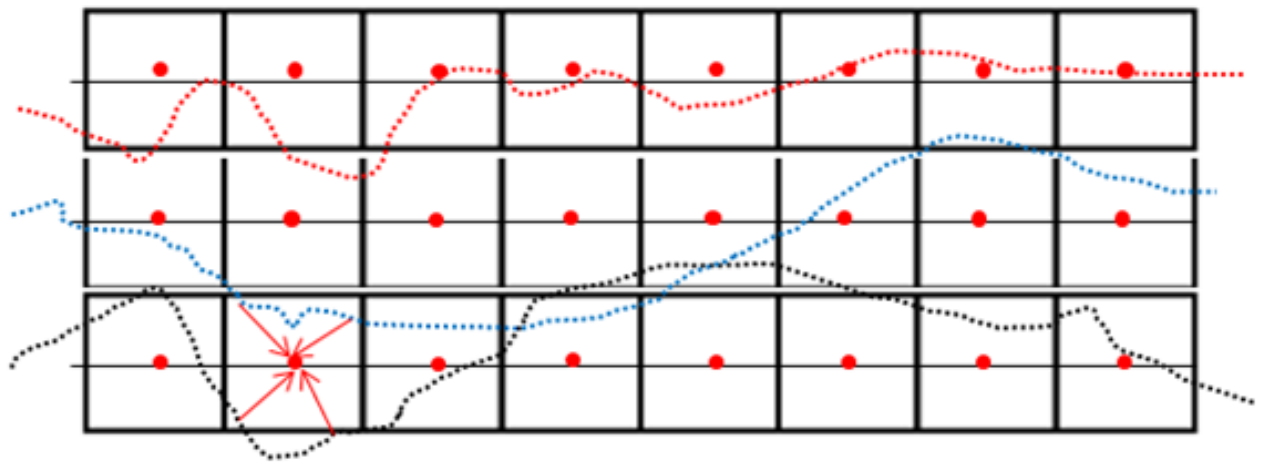

Figure 6 Actual GPR survey traces and interpolated rectangular grid points.

\section{FIELD EVALUATION}

Tohoku University and University of Miami are collaboratively working on the application of 3DGPR for detection of buried explosive devices. Currently we are investigating the use of iGPS, a large work volume metrology method, as a complementary tracking device for the CCD camera, which has been used in ALIS. iGPS can provide absolute and better than centimetre precise $\mathrm{x}, \mathrm{y}, \mathrm{z}$ coordinates to multiple mine sensors at the same time. At University of Miami we have developed a novel 3DGPR system for efficient and high-resolution 3D shallow subsurface scanning of larger areas (25 $\mathrm{m}^{2}$ to thousands of square meters) with irregular topography [8].

Tohoku University and University of Miami have conducted the first field measurements, and found that the 3DGPR system can visualize small buried low-metal landmines with high resolution. Fig.7 shows the test site and iGPS transmitter set for 3DGPR data acquisition. The site area is $8 \mathrm{~m} \times 10 \mathrm{~m}$. 


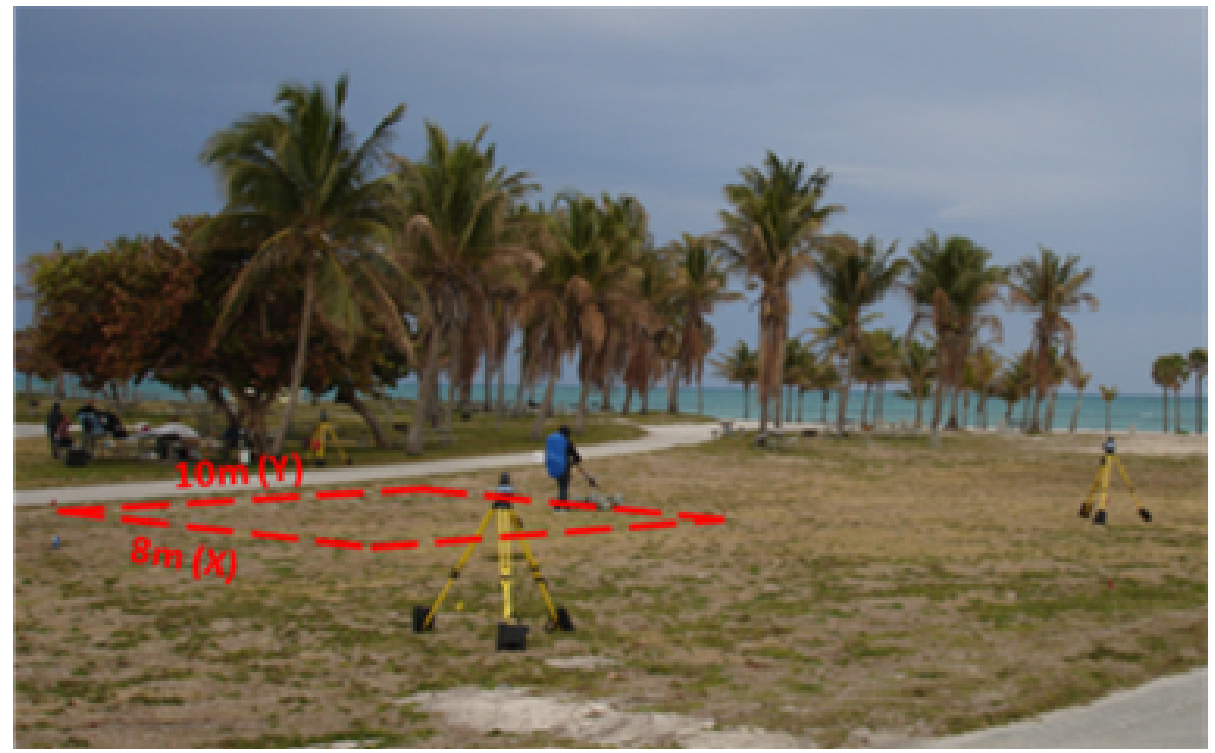

Figure 7 Test site for 3DGR applied to mine detection.

The soil in the site is sandy, but contained inhomgoneneous humidity. Plastic land mine models (PMN-2 and Type 72) were buried with different depths $(0 \mathrm{~m}, 5 \mathrm{~m}, 10 \mathrm{~m}$, and $15 \mathrm{~m})$ as well as with two positions, namely horizontal and $45 \mathrm{deg}$ inclination as shown in Fig.8.

A 500MHz commercial GPR system (Mala Geoscience, RAMAC GPR) was used for this measurement. The data was acquired along survey lines having a $5 \mathrm{~cm}$ spacing.

In this system, position data by iGPS and GPR are stored independently, and data will be merged when the data will be processed. Interpolation of data was carried out and the GPR data was re-arranged on rectangular grids of $25 \mathrm{~mm} \mathrm{x}$ $25 \mathrm{~mm}$. Other data position corrections have also been implemented.

Fig.9 and 10 show examples of the processed GPR horizontal profiles. Land mine models having less than $10 \mathrm{~cm}$ diameter (PMN-2 and Type-72) could be clearly imaged.

Fig. 11 shows one of the 3D displays of the processed GPR signal. Both horizontal and inclined mines are clearly imaged. Note that this GPR profile is not processed, but the raw GPR image 


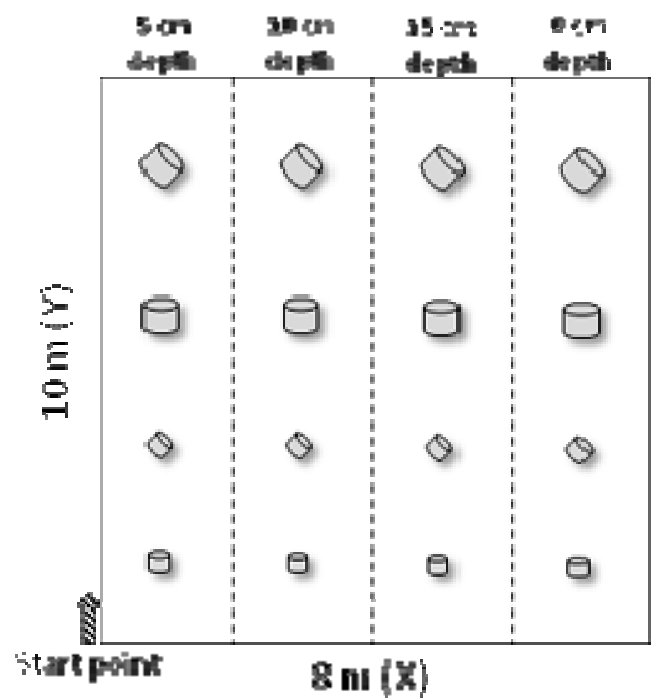

Figure 8 Layout of the buried landmines.

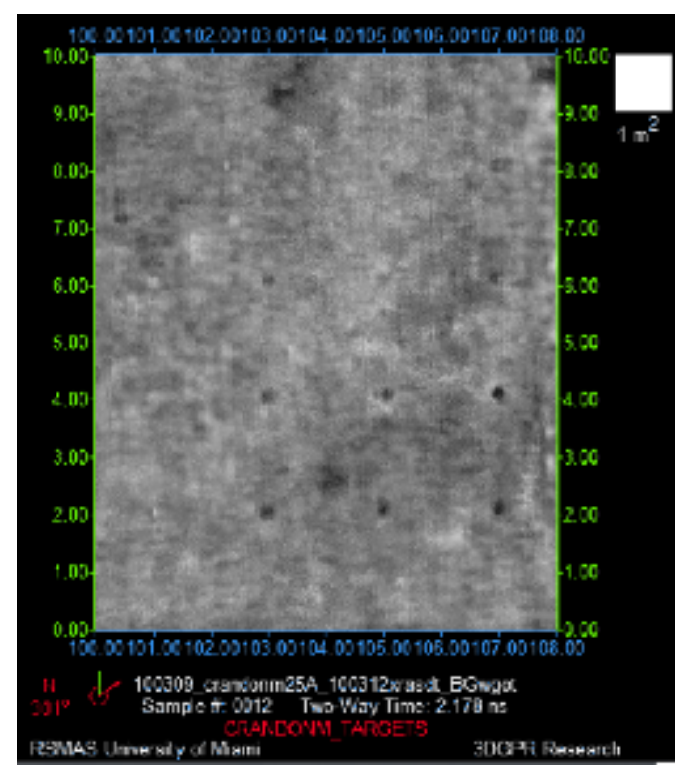

Figure 9 Landmines with depth ranges from 5 to $15 \mathrm{~cm}$ imaged by 3DGPR. 

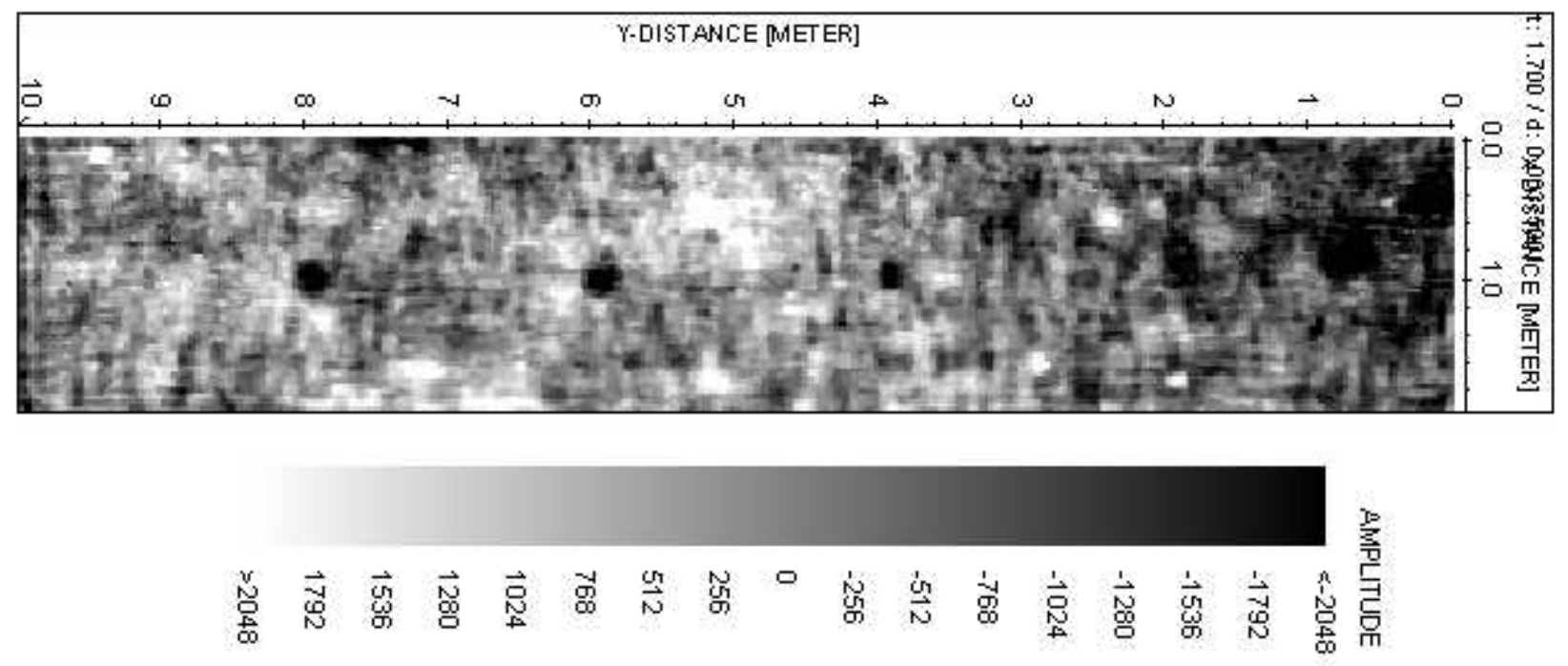

Figure.10 A horizontal slice image at $15 \mathrm{~cm}$ obtained by 3DGPR. Data acquired on a $25 \mathrm{x} 25 \mathrm{~mm}$ grid with a $500 \mathrm{MHz}$ GPR antenna positioned with IGPS. From rigght, 45-degree Type-72, Horizontal Type-72, 45-degree PMN-2 and Horizontal PMN-2 are buried.

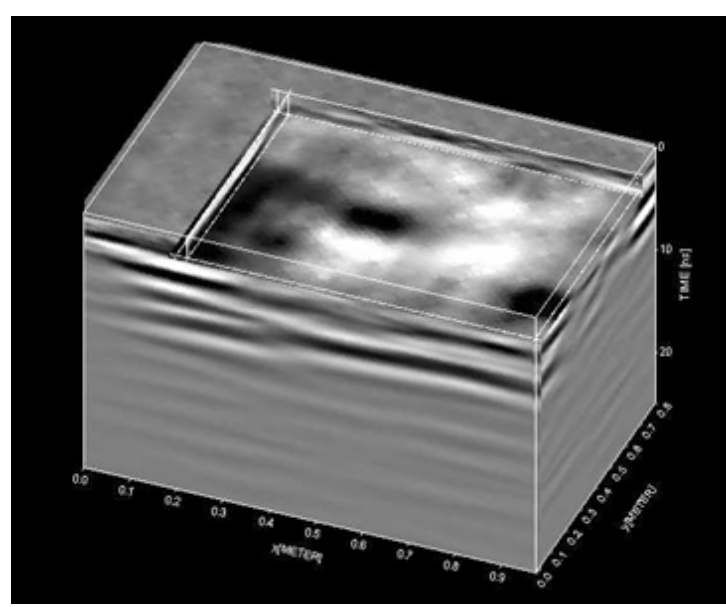

(a) 0-degree (Horizontal)

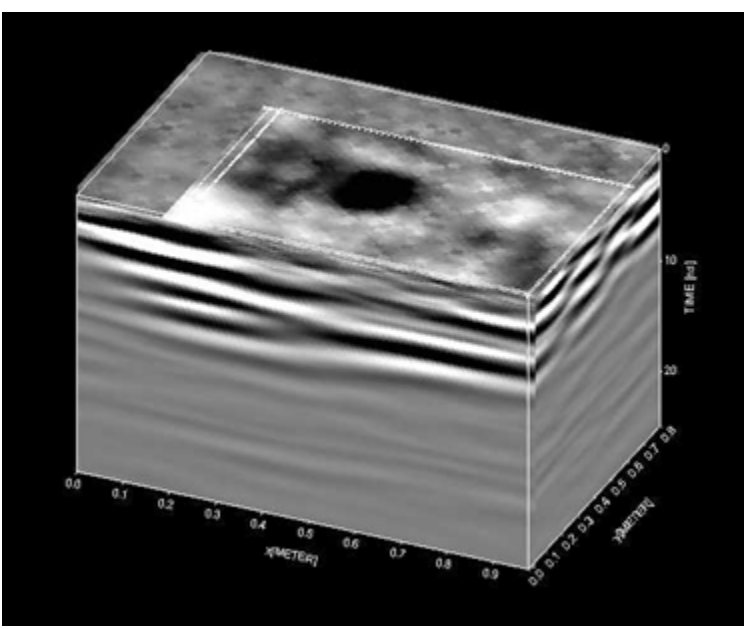

(b) 45-degree inclined

Figure.11 3DGPR Image of an $80 \mathrm{~mm}$ diameter low metal Type-72 mine inclined 45 degree at the depth of $5 \mathrm{~cm}$. Data acquired on a $25 \times 25 \mathrm{~mm}$ grid with a $500 \mathrm{MHz}$ GPR antenna positioned with IGPS. The top surface of the displayed 3D cube measures $1000 \times 800$ $\mathrm{mm}$.

We used a "500MHz" GPR antenna, which operates at the frequency bandwidth effectively about $100 \mathrm{MHz}$ and the center frequency is at $500 \mathrm{MHz}$. The range resolution is about $10 \mathrm{~ns}$ pulse width, and the spatial duration of the pulse is approximately $1 \mathrm{~m}$, by assuming the dielectric permittivity of the soil as 9 . The imaged shape clearly shows the cylindrical structure of model land mines, and we think we could demonstrate that 3DGPR system can provide very accurate images. 


\section{CONCLUSION}

The initial field tests show that the combination of CCD camera local sensor tracking with large work volume iGPS has the potential to deliver the centimetre resolution ground surface and subsurface images necessary to find small low-metal content AP landmines. With such a combined tracking solution, scan data acquired simultaneously by multiple demining teams working at a mine polluted site can be geo-referenced in real time and used for integrated target analysis.

\section{ACKNOWLEDGEMENTS}

This work was supported by JST Japan - U.S. Joint Research Program "Advanced Integrated Sensor Technology" and JSPS Grant-in-Aid for Scientific Research (S) 18106008, (A) 23246076

\section{REFERENCES}

[1] http://www.jst.go.jp/kisoken/jirai/index-e.html

[2] M. Sato, J.Fujiwara and K.Takahashi, "The Development of the Hand Held Dual Sensor ALIS," Proc. Detection and remediation technologies for mines and minelike targets X II, Proc. SPIE, 6553, 65531C-1-65531C10(2007).

[3] Motoyuki Sato, Takao Kobayashi, Kazunori Takahashi, Jun Fujiwara, Xuan Feng , "Vehicle mounted SARGPR and its evaluation," Defense and Security Symposium,(2006).

[4] http://www.alis.jp/

[5] Grasmueck, M. and D.A. Viggiano, "Integration of Ground-Penetrating Radar and Laser Position Sensors for Real-Time 3D Data Fusion," IEEE Transactions on Geoscience and Remote Sensing, 45(1), 130-137 (2007)

[6] M. Sato, J.Fujiwara and K.Takahashi, "The Development of the Hand Held Dual Sensor ALIS," Proc. Detection and remediation technologies for mines and minelike targets X II, Proc. SPIE, 6553, 65531C-1-65531C10.(2007).

[7] Motoyuki Sato, Takao Kobayashi, Kazunori Takahashi, Jun Fujiwara, Xuan Feng, "Vehicle mounted SARGPR and its evaluation,".Defense and Security Symposium,(2006)

[8] M. Sato, A. Gaber, Y. Yokota, M. Grasmueck and P. Marchesini, "CCD Camera and IGPS Tracking of Geophysical Sensors for Visualization of Buried Explosive Devices," Proceeding of the International Conference on Indoor Positioning and Indoor Navigation (IPIN), IEEE Xplore, 974-978 (2010).

[9] Motoyuki Sato . Yuya Yokota. Kazunori Takahashi, "ALIS : GPR System for Humanitarian Demining and Its Deployment in Cambodia," Journal of the Korean Institute of Electromagnetic Engineering and Science, 12(1), (2012). 